J. Phys. IV France 1 (2018)

$\operatorname{Pr} 1-1$

(c) EDP Sciences, Les Ulis

\title{
GAIA Spectroscopy and Radial Velocities
}

\author{
Ulisse Munari ${ }^{1}$ \\ ${ }^{1}$ Osservatorio Astronomico di Padova, Sede di Asiago, I-36012 Asiago (VI), Italy
}

\begin{abstract}
GAIA spectroscopic and radial velocity performancies are reviewed on the base of groundbased test observations and simulations. The prospects for accurate analysis of stellar atmospheres (temperature, gravity, chemical abundances, rotation, peculiarities) and precise radial velocities (single stars, binaries, pulsating stars) are colorful provided the spectral dispersion is high enough. A higher dispersions also favors a given precision of radial velocities to be reached at fainter magnitudes: for example, with current parameters for GAIA spectrograph, a $1 \mathrm{~km} \mathrm{sec}^{-1}$ accuracy on epoch RVs of a K0 star is reached at $V \sim 13.0$ mag with $0.25 \AA /$ pix dispersion spectra, at $V \sim 10.3 \mathrm{mag}$ for $0.5 \AA /$ pix, and $V \sim 6.7 \mathrm{mag}$ for $1 \AA /$ pix. GAIA radial velocities for single stars can match the $\sim 0.5 \mathrm{~km} \mathrm{sec}^{-1}$ mean accuracy of tangential motions at $V=15$ mag, provided the observations are performed at a dispersion not less than $0.5 \AA /$ pix.
\end{abstract}

\section{INTRODUCTION}

The giant leap that GAIA spectroscopy will lead us through can be sized by four basic considerations: (a) GAIA will record multi-epoch spectra for a magnitude complete sample of stars $\sim 10^{3}$ larger than any whole-sky existing database (e.g. HD survey, progressing Michigan project, etc.); (b) for each target, an average of 67 epoch spectra will be recorded over the five year mission lifetime; $(c)$ the wavelength and flux calibrated spectra will be available in digital format to the community; $(d)$ the foreseeable spectral dispersion $(0.75 \AA /$ pix are currently baselined) is significantly higher that those of other whole-sky surveys.

A review of GAIA spectroscopy has already been presented by Munari (1999a, hereafter M99a). We will consider here mainly updates to the content of M99a reflecting advancements in some areas over the last couple of years. Therefore, to cope with the generous but limited amount of space available to this review in its printed format, basic physics and overall considerations developed in M99a will not be discussed here again. Technical aspects connected to spacecraft optical and mechanical assembly, telemetry budgets, modus operandi, limiting magnitudes etc., are covered in the ESA's GAIA Concept and Technology Study Report (ESASCI-2000-4) and in abridged format in Perryman et al. (2001).

GAIA spectra will cover the 8490-8750 $\AA$ wavelength range, centered on the near-IR Ca II triplet and head of the Paschen series. The extention to $8750 \AA$ (the redder Ca II line laying at $8662.141 \AA$ ) allows observation of remarkable N I \#1 and 8 multiplets in hot stars and particularly strong Fe I, Mg I and Ti I lines in cool stars. GAIA wavelength range is the only spectral window in the near-IR which is not crunched by telluric absorptions (cf. Figure 1 of M99a), allowing uncontaminated ground-based preparatory and follow-up observations. 
Ca II triplet is by far the strongest line feature in the red/near-IR spectra of cool stars (cf. Fig 1 of Munari \& Castelli 2000; Jaschek \& Jaschek 1995), being core-saturated even in metal-poor halo stars, thus allowing derivation of radial velocities on epoch-spectra of even the faintest and more metal deprived GAIA spectral targets. Cool stars will vastly dominate among the latter (with $\mathrm{O}$ and $\mathrm{B}$ stars barely traceable). GAIA wavelength range (restricted to $\triangle \lambda \sim 250 \AA$ by optical and telemetry constraints) is placed toward peak emission in cool stars and lower interstellar extinction, with obvious benefits for the number of detectable targets.

The number of GAIA spectral targets (approaching $10^{8}$ in current estimates), will require fully automatic data treatment and analysis. Line intensities and ratios may be useful in preflight ground-based preparatory work and/or quick-look classification and re-direction along the reduction pipeline of actual GAIA data. However, it is clear that proper exploitation of GAIA spectra will required a smart spectral synthesis approach. Even if currently available synthetic models of stellar atmosphere (MARCS, ATLAS, Phoenix, etc.) and nebular regions (CLOUDY, etc.) will be probably quite improved by the time GAIA data will be available (and new families of models will probably be developed too), nevertheless they play a fundamental role right now in the current infancy of GAIA spectroscopy, by offering precious guidelines, ways to improve basic underlying physics (for ex. atomic constants) and unlimited databases for simulations.

Most of GAIA performances will depend on the eventually adopted spectral dispersion. An example of how lowering the resolution affects spectral appearance of a K0 III star - a typical GAIA target - is given in Figure 1. On one side the race for fainter limiting magnitudes and smallest demand on telemetry push for mid to low spectral dispersions. On the other side, getting the best astrophysical return and the highest accuracy of radial velocities most decidedly ask for high dispersions. The best compromise will have to balance between them.

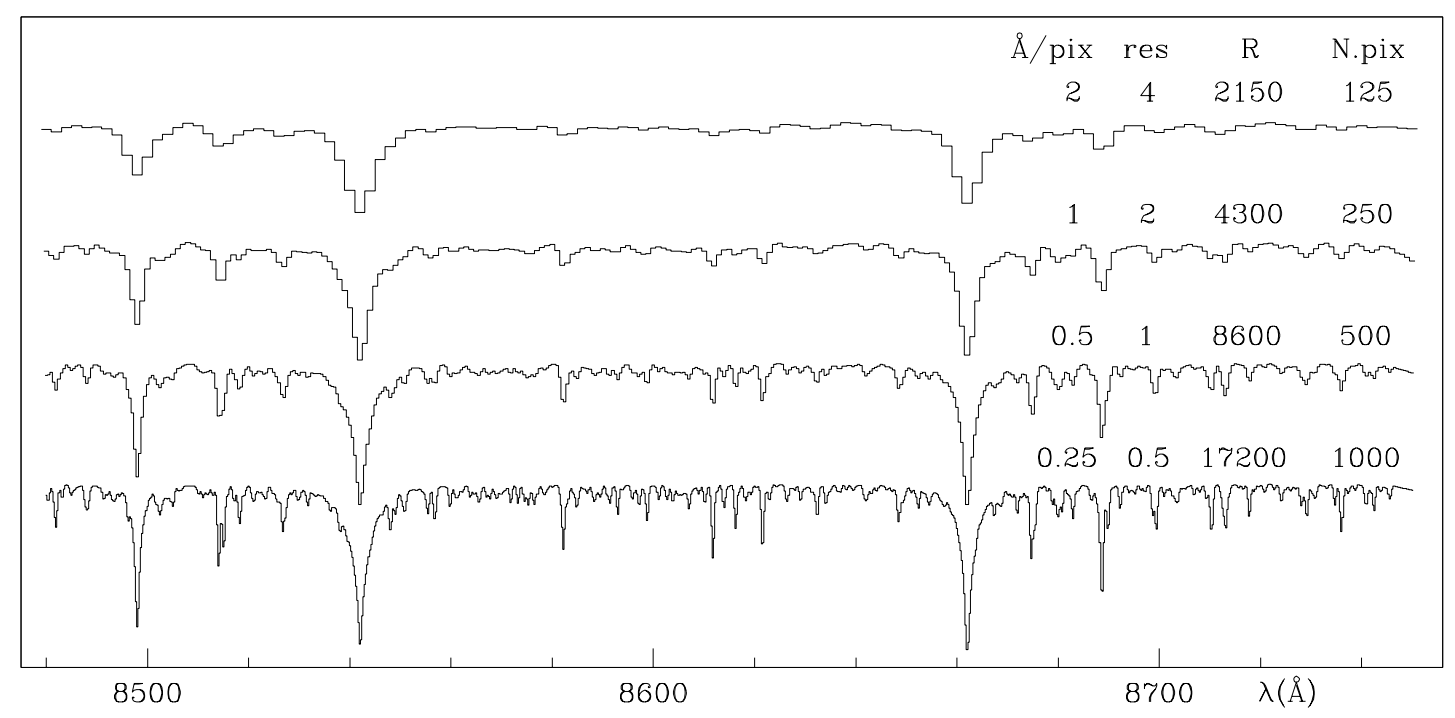

Fig. 1. The same K0 III spectrum at different dispersions/resolutions. The number in the four columns give the dispersion (in $\AA$ /pix), the resolution (in $\AA$, with $\operatorname{FWHM(PSF)}=2$ pix), the resolving power and the number of pixel required to cover the whole GAIA wavelength range. 


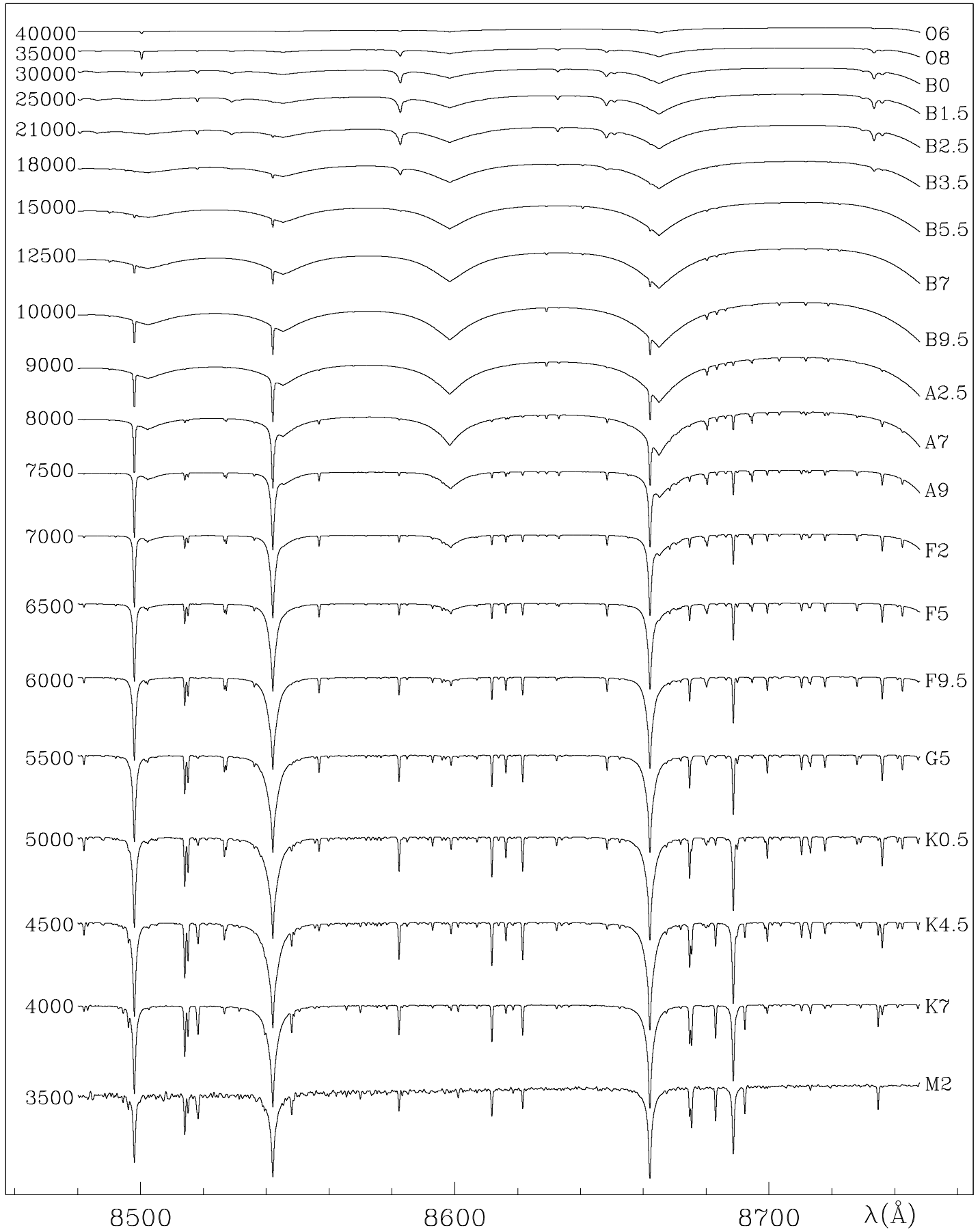

Fig. 2. Sequence of synthetic spectra (from Munari \& Castelli 2000, Castelli \& Munari 2001) illustrating the variations along the main sequence $\left(T_{e f f}\right.$ in $\mathrm{K}$ on the left and corresponding spectral type for luminosity class $\mathrm{V}$ on the right) for moderately metal poor stars $\left(\left[\mathrm{Z} / \mathrm{Z}_{\odot}\right]=-0.5\right)$. All spectra are on the same ordinate scale, only displaced in their zero-points. 
The spectra presented in this review carry a $0.25 \AA /$ pix dispersion, at the high end of the $0.25-1.0 \AA /$ pix range currently considered, thus allowing the reader to guess the highest possible GAIA performancies. In the following, effective temperatures and surface gravities for MKK spectral types are adopted from Strayzis (1992).

\section{STELLAR PHYSICAL AND CLASSIFICATION PARAMETERS}

\subsection{Temperature/spectral type}

A temperature sequence spanning the MKK classification scheme is presented in Figure 2. M to F spectral types are governed by the Ca II triplet, hotter ones by He I, N I and the hydrogen Paschen series. A rich forest of metal lines populates the GAIA wavelength range (cf. Figure 3 in M99a), which is dominated by Ca II, Fe I, Ti I atomic lines and CN molecular transitions. Relevant absorptions are also due to Mg I, Si I, Cr I, N I, Co I, Ni I, Mn I, S I as well as TiO, with other elements and molecules contributing weaker spectral signatures.

Such a harvest makes spectral classification over the $\Delta \lambda \sim 250 \AA$ GAIA range nearly as much easy as it is for the $\triangle \lambda \sim 1000 \AA$ classical MKK range (which extends from 3900 to $4900 \AA$ ). Only O and B stars perform less good, which is however of no concern given their barely traceable fraction among GAIA targets.

Diagnostic line ratios useful for spectral classification purposes can be easily derived on GAIA spectra. Two examples of line ratios are illustrated in Figure 3. Near-IR Ca II over Paschen lines are highly effective in classifying late B, A and F stars, as it if for Ca II H and $\mathrm{K}$ over Balmer lines in optical spectra. Ti $8674.7 / \mathrm{FeI} 8675.4$ works very well in G, K and M stars, with the following expression giving the fitting curve in Figure 3 (right panel):

$$
R=\frac{\operatorname{TiI} 8674.7 \AA}{\mathrm{FeI} 8675.4 \AA} \Longrightarrow \quad T_{\text {eff }}=6072-2188 \times R+364 \times R^{2}
$$

A $\triangle R=4 \%$ error in the line ratio (typical for the observations in Figure 3) corresponds to just $\triangle T_{\text {eff }}=65 \mathrm{~K}$. It worth to mention that also GAIA photometry will (obviously) estimate the temperature of target stars (however with contamination from interstellar reddening, if present), providing independent data to be compared with spectroscopic findings.

\subsection{Gravity/luminosity class}

Figure 5 illustrates line behavior with luminosity class. With lowering surface gravity (increasing luminosity, decreasing pressure) the intensity of absorption lines goes up, as much as in classical optical spectra and required by physics. The equivalent width of Ca II lines shows a pronounced positive luminosity effect (collisional de-population of excited state less effective with decreasing pressure). Width of Paschen lines presents a negative luminosity effect (being pressure broadened, similarly to Balmer lines) as given in Figure 4. It is again worth noticing that surface gravities will be also quite effectively measured by GAIA combining astrometric distances with photometry (cf. sect. 2.1 in Munari 1999b, hereafter M99b).

As for temperature/spectral type, it is easy to derive diagnostic line ratios highly sensitive to surface gravity/luminosity class. The steep behavior of (Si I 8728.0 + Fe I 8729.1)/(Mg I 8736.0) in G5 stars is illustrated in Figure 4, with fitting curve given by:

$$
R=\frac{\operatorname{SiI}+\mathrm{FeI} 8728 \AA}{\operatorname{MgI} 8736 \AA} \Longrightarrow \quad \lg g=3.6+3.0 \times R-2.57 \times R^{2}
$$

A $\triangle R=3 \%$ error (typical for Figure 4 data) corresponds to just $\triangle \lg g=0.11$ 

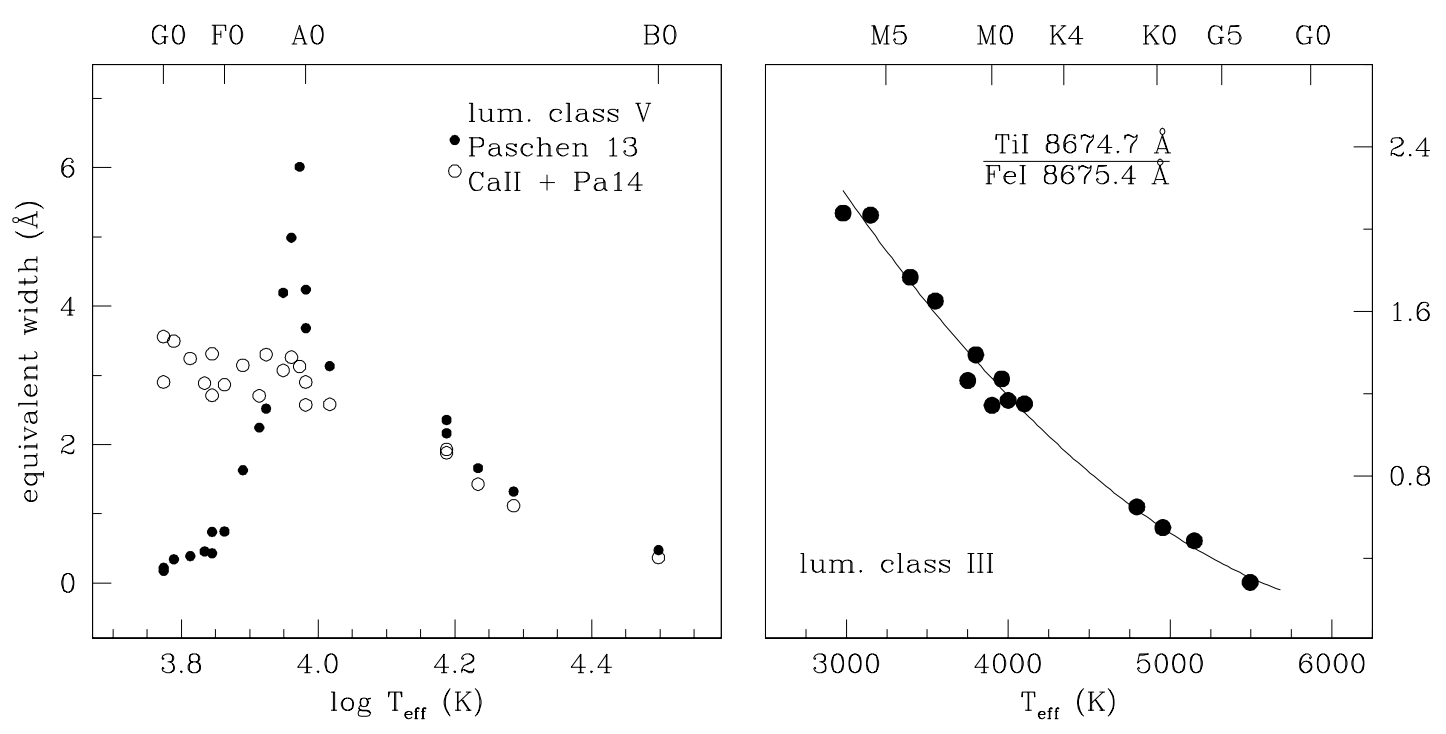

Fig. 3. Example of temperature diagnostic ratios (Boschi 2000, Boschi \& Munari 2001) from real spectra (from Munari \& Tomasella 1999). Left panel: hydrogen Paschen and Ca II lines in main sequence stars. Right panel : a powerful temperature diagnostic ratio for cool giants (requiring 0.25 $\AA /$ pix dispersion spectra to resolve the lines)
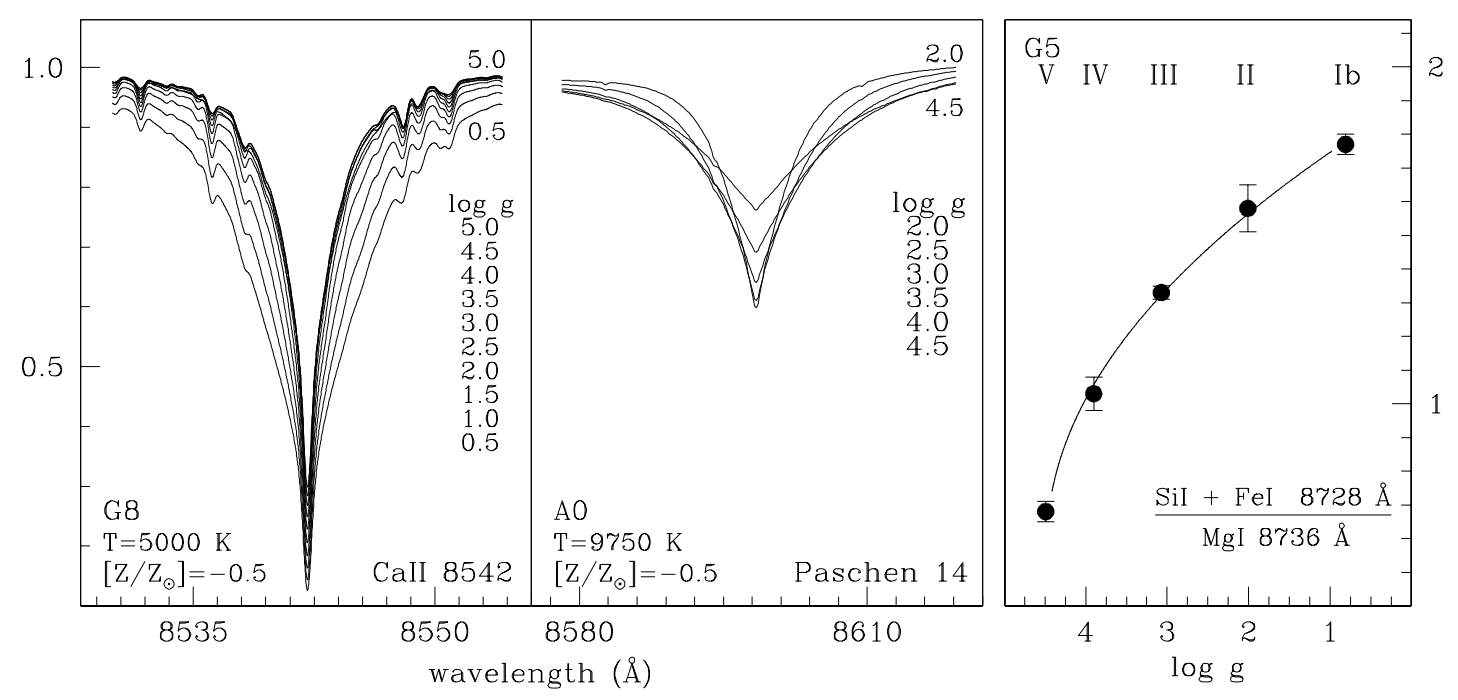

Fig. 4. Left: gravity effects on the profile of CaII $8542 \AA$ for $T_{e f f}=5000 \mathrm{~K}$ ( $\sim$ G8 spectral type) and $\left[\mathrm{Z} / \mathrm{Z}_{\odot}\right]=-0.5$ (synthetic spectra from Munari \& Castelli 2000). Center: gravity effects on the profile of Paschen $14,8598 \AA$ for $T_{\text {eff }}=9750 \mathrm{~K}$ ( $\sim \mathrm{A} 0$ spectral type) and $\left[\mathrm{Z} / \mathrm{Z}_{\odot}\right]=-0.5$ (synthetic spectra from Zwitter et al. 2001). Right: dependence upon gravity of the ratio (SiI 8728.0 + FeI 8729.1)/MgI 8736.0 (Boschi and Munari 2001) on G5 real spectra (from Munari \& Tomasella 1999). 


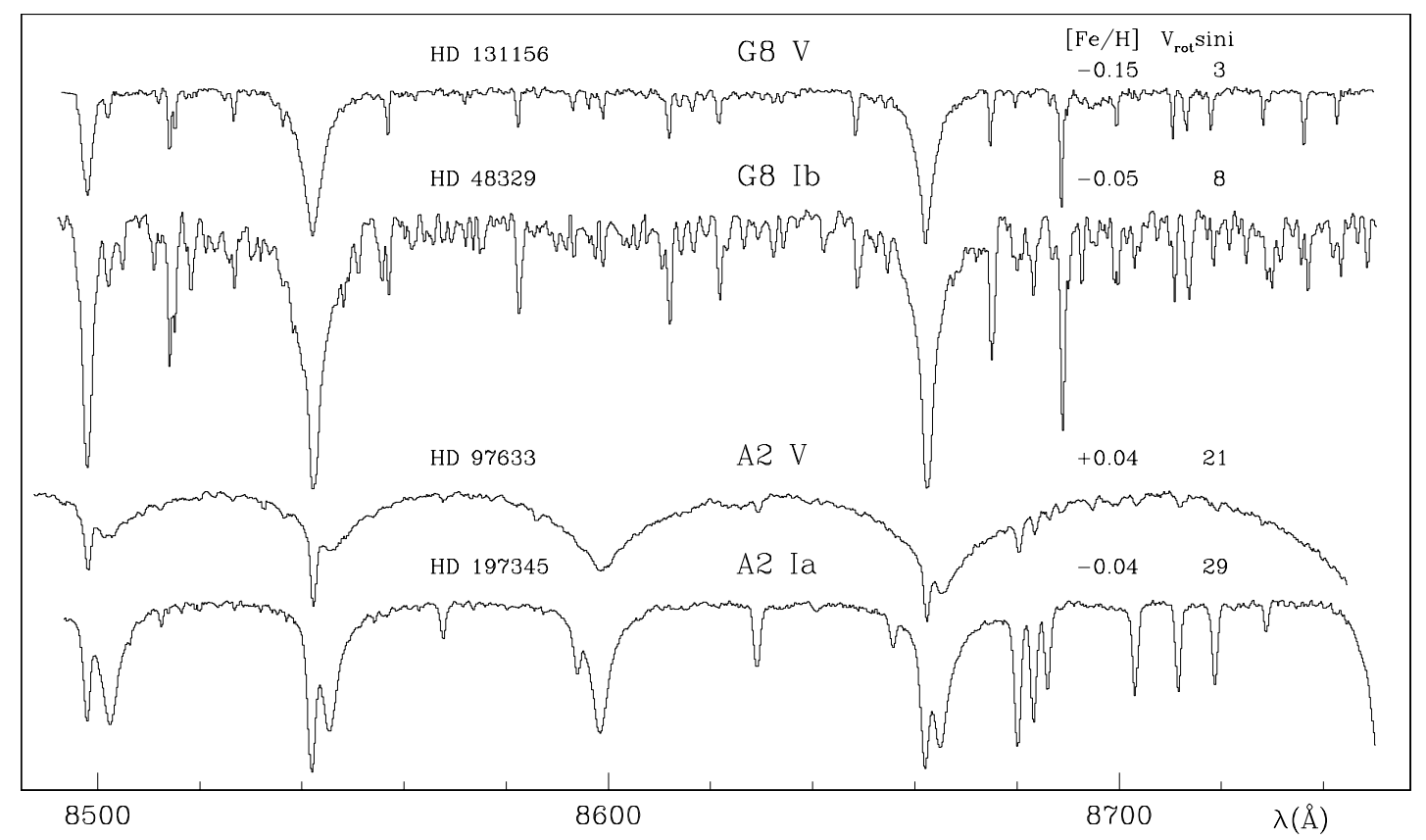

Fig. 5. Left: gravity effects on G8 and A2 spectra (from Munari \& Tomasella 1999).

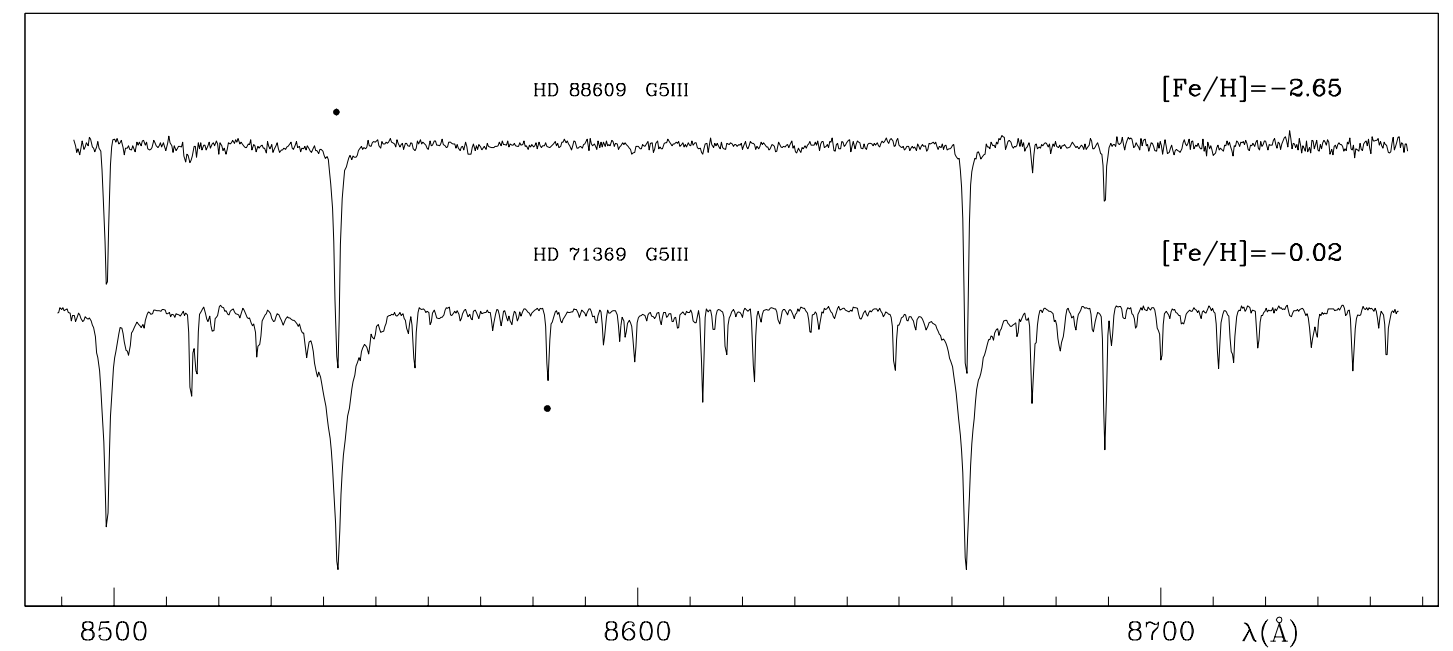

Fig. 6. Examples of G5 III stars of quite different metallicities (from Marrese 2000). On cool stars, even at very low abundances, Ca II lines remain strong, allowing accurate radial velocity measurements, while nearly all other metallic lines have gone. 


\subsection{Chemical abundances}

The intensity of absorption lines obviously correlates with the chemical abundances. Figure 6 presents spectra of two G5 III stars of widely differing metallicities $([\mathrm{Fe} / \mathrm{H}]=-2.65$ and -0.02$)$.

Even at the lowest metallicities (like those found in the Halo and globular clusters), Ca II lines remain core-saturated while nearly all other metallic lines have gone, allowing accurate radial velocity measurements and fine rotational velocity estimates.

The possibility to perform chemical analysis on the recorded spectra will depend on the spectral dispersion that will be eventually adopted for GAIA. Figure 1 is illuminating in this sense. At $0.25 \AA /$ pix hundreds of absorptions lines blossom over the whole GAIA range, tracing tens of different elements which individual chemical abundances can be derived as routinely done with high resolution optical spectra secured with ground-based telescopes. At $0.5 \AA /$ pix, lines from different elements merge into blends and individual chemical abundances can be derived (with a limited accuracy) probably only for Fe I, Ti I and Mg I (tests are underway). At even lower dispersions, chemical analysis looks hopeless, with absorption lines washing into an unfeatured continuum.

It is also worth to mention that GAIA narrow band photometry (M99b; V.Vansevicius, M.Grenon, these proceedings) should estimate metallicity (a weighted average of individual chemical abundances) from color indexes with a sensivity comparable to best performing, existing ground-based photometric systems (Moro \& Munari 2000).

\section{ROTATIONAL VELOCITIES}

Projected rotational velocity $\left(V_{\text {rot }} \sin i\right)$ is another intrinsic property of stars that GAIA can measure with relevant confidence, provided the spectral dispersion is high enough. The impact of axial rotation on stellar evolution is only recently being appreciated and modeled (cf. A.Maeder, this volume; Maeder \& Meynet 2001 and references therein).

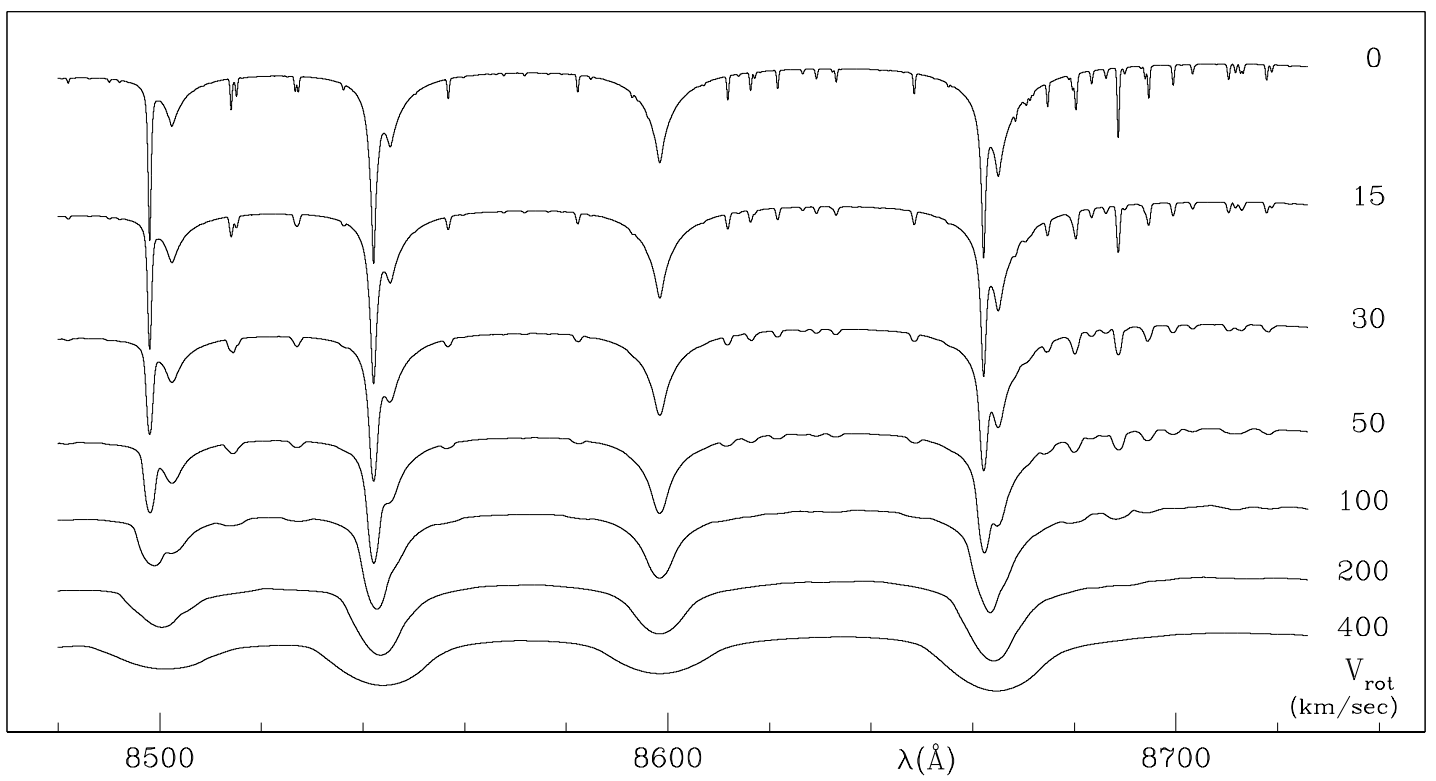

Fig. 7. Rotational velocity sequence for F0 III giants (spectra from Zwitter et al. 2001). 
A sequence of rotationally broadened F0 III spectra is presented in Figure 7. Ca II and Paschen lines perform equally well up to very high $V_{\text {rot }}$, offering bright prospects for measuring rotational velocities both in cool and hot stars, respectively. Other, weaker lines are useless at $V_{\text {rot }} \geq 40 \mathrm{~km} \mathrm{sec}^{-1}$, being washed out in the adjacent continuum.

Rotational velocities of G-K-M stars (constituting the largest fraction of GAIA targets) are usually confined to $V_{\text {rot }} \leq 15 \mathrm{~km} \mathrm{sec}^{-1}$ (Glebocki et al. 2000). To effectively discriminate at so low values, a properly high spectral resolution is necessary. $0.5 \AA$ /pix spectra would not allow GAIA to distinguish $V_{\text {rot }} \sin i=15 \mathrm{~km} \mathrm{sec}^{-1}$ stars from non-rotating ones, i.e. rotational velocities will be undetermined for the majority of GAIA targets. Instead, $0.25 \AA /$ pix spectra can detect and measure rotational velocities to an accuracy of $\triangle V_{\text {rot }} \sin i=5 \mathrm{~km} \mathrm{sec}^{-1}$, thus providing decent sensitivity to rotation for all GAIA targets. The rotational broadening of narrow lines may be expressed in term of the total width at half intensity (in $\AA$ ) as

$$
V_{\text {rot }} \sin i=42.4 \times H I W-35 \quad \mathrm{~km} \mathrm{sec}^{-1}
$$

\section{RADIAL VELOCITIES}

\subsection{Single stars}

Munari et al. (2001a, hereafter M01a) have investigated in detail GAIA radial velocity performances as function of spectral resolution and signal-to-noise ratio, by obtaining 782 real spectra and using them as inputs for 6700 automatic cross-correlation runs. M01a have explored the dispersions $0.25,0.5,1$ and $2 \AA$ /pix (bracketing the $0.75 \AA$ /pix currently baselined) over S/N ranging from 12 to 110, carefully maintaining the condition FWHM (PSF) $=2$ pixels. M01a have investigated late-F to early-M stars (constituting the vast majority of GAIA targets), slowly rotating ( $<V_{\text {rot }} \sin i>=4 \mathrm{~km} \mathrm{sec}^{-1}$, as for field stars at these spectral types), of solar metallicity $(<[\mathrm{Fe} / \mathrm{H}]>=-0.07)$ and not binary (target stars selected among IAU standard radial velocity stars). The results are accurately described by:

$$
\lg \sigma=0.6 \times\left(\lg \frac{S}{N}\right)^{2}-2.4 \times \lg \frac{S}{N}+1.75 \times \lg D+3
$$

where $\sigma$ is the cross-correlation standard error (in $\mathrm{km} \mathrm{sec}^{-1}$ ) and $D$ is the spectral dispersion (in $\AA /$ pix). Inserting $0.25,0.5,1,2$ for $D$ in the above expression gives the four fitting curves in Figure 8. The spectral dispersion is the dominant factor governing the accuracy of radial velocities, with $\mathrm{S} / \mathrm{N}$ being less important. Spectral mis-match affects the results only at the highest S/N. M01a findings suggests that mission-averaged GAIA radial velocities on nonvariable, single stars can match the $\sim 0.5 \mathrm{~km} \mathrm{sec}^{-1}$ mean accuracy of tangential motions at $V=15 \mathrm{mag}$, provided the observations are performed at a dispersion not less than $0.5 \AA /$ pix. Binary and/or fast rotating and/or pulsating and/or surface spotted stars will require higher accuracies (thus higher spectral dispersions) in order to disentangle perturbing effects from baricentric motion.

The flattening of RV performances at the highest $\mathrm{S} / \mathrm{N}$ in Figure 8 and Eq.(4.1) results from the spectral type mismatch between template and program stars in M01a study (while metallicities and rotational velocities were instead pretty similar and therefore not influent). The large but however limited number of real spectra (782) and the extreme paucity of IAU RV standards sharing the same spectral classification, forced spectral type mismatch in M01a investigation. Extensive simulations on huge numbers of synthetic spectra have been performed by Zwitter (2001) to get rid of mismatches unavoidable with real spectra. His results confirm Eq. (4.1) and Figure 8 behavior at low and mid S/N. As expected, they do not show the 


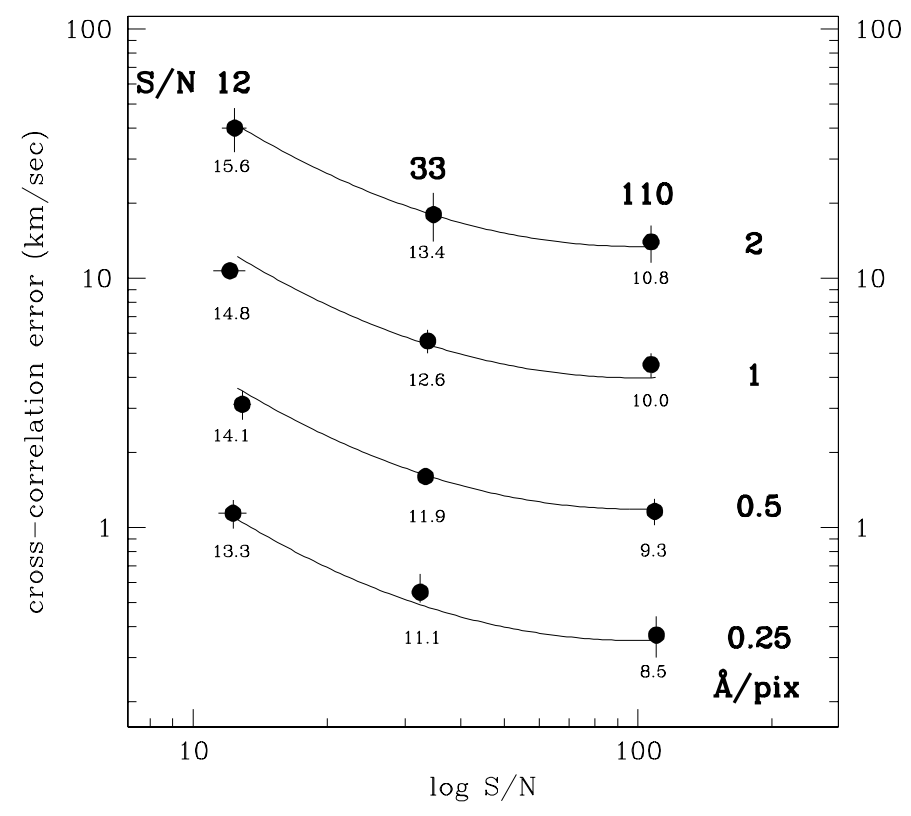

Fig. 8. The accuracy of radial velocities obtained via crosscorrelation for late- $\mathrm{F}$ to early-M stars as function of $\mathrm{S} / \mathrm{N}$ and spectral dispersion (Agnolin 2000; $\mathrm{Mu}-$ nari et al. 2001a). The results based on 782 real spectra and 6700 cross-correlation runs include the effect of mis-match between object and template stars (removing it would reduce the upward curvature at higher $\mathrm{S} / \mathrm{N})$. The $V$ magnitude of unreddened G5 $\mathrm{V}$ stars producing epoch spectra of the given $\mathrm{S} / \mathrm{N}$ is reported next to the points (computed for: mirror size $=75 \times 70 \mathrm{~cm}$; overall throughput $=35 \%$; crossing time $=60.8 \mathrm{sec} ; I_{\mathrm{C}}^{\operatorname{mag}=0.0}=$ $1.196 \times 10^{-9} \mathrm{erg} \mathrm{cm}^{-2} \mathrm{sec}^{-1} \AA^{-1}$ $=520$ photons $\mathrm{cm}^{-2} \mathrm{sec}^{-1} \AA^{-1}$ ; R.O.N. $=3 e^{-1}$; dark $=0.01$ $e^{-1} \sec ^{-1} ;$ background $I_{\mathrm{C}}=21.5$ $\left.\operatorname{arcsec}^{-2}\right)$.

upward curvature at the highest $\mathrm{S} / \mathrm{N}$ induced in Figure 8 by the spectral mismatch (see also D. Katz, these proceedings, for additional results on GAIA radial velocities).

M01a and Zwitter (2001) investigations argue in favor of increasing the spectral dispersion to get more accurate radial velocities, for a fixed photon budget (like in GAIA fixed exposure time observations). From the results with real spectra of Figure 8, with current parameters for GAIA spectrograph, a $1 \mathrm{~km} \mathrm{sec}^{-1}$ accuracy on epoch RVs of a K0 star is reached at $V \sim 13.0$ mag with $0.25 \AA /$ pix spectra, at $V \sim 10.3 \mathrm{mag}$ for $0.5 \AA / \mathrm{pix}$, and $V \sim 6.7 \mathrm{mag}$ for $1 \AA / \mathrm{pix}$ dispersion. The reason for this is quite obvious looking at Figure 1: at high dispersions all pixels carry RV information, even at low $\mathrm{S} / \mathrm{N}$, while at low dispersion only a few pixels carry $\mathrm{RV}$ information, no matter how high is the $\mathrm{S} / \mathrm{N}$.

\subsection{Binary stars}

Eclipsing stars represent the most astrophysically relevant type of binary stars GAIA will spectroscopically observe during its mission.

They are a prime tool to derive fundamental stellar parameters like mass and radius, or the temperature scale. Moreover, their use as accurate, geometric distance indicators is rapidly growing (current best distances to LMC, dwarf spheroidals, globular clusters). Their study is by no means a simple task as evidenced by the fact that stellar parameters have been derived with an accuracy of $1 \%$ or better for less than a hundred objects. Scaling the Hipparcos results ( $0.8 \%$ of the 118218 stars surveyed turned out to be eclipsing), $\sim 4 \cdot 10^{5}$ of all $V \leq 15$ mag GAIA targets should be eclipsing binaries, with a average G7 spectral type. It may be estimated that about $25 \%$ of the eclipsing binaries will be double-lined in GAIA spectral observations (cf. Carquillat et al. 1982), thus $\sim 1 \cdot 10^{5}$ of all $V \leq 15$ mag GAIA targets. Even if for only $5 \%$ 
of them it should be possible to derive orbits and stellar parameters at $1 \%$ precision, this still would be $100 \times$ what humanity have so far collected from devoted ground-based efforts during the whole last century (cf. Andersen 1991).

That a $1 \%$ accuracy in orbital and physical parameters of eclipsing binaries is feasible within GAIA has been demonstrated by Munari et al. (2001b, hereafter M01b). $0.25 \AA /$ pix spectra over the GAIA range have been secured from the ground for 15 eclipsing binaries (mostly unstudied in literature and distributed among detached, semidetached, contact and intrinsic variable types) and combined with Hipparcos $V_{T}, B_{T}, H_{P}$ photometry to properly simulate GAIA data harvest.

Example results from M01b for V505 Per are given in Figure 9. Semi-major axis, masses, surface gravities, effective temperatures, inclination, eccentricity, baricentric velocity, epoch and orbital period are in the $1 \%$ or better accuracy regime. Radii of individual components (and therefore individual bolometric magnitudes) however depends on how well the branches of eclipses are mapped. In the case of Hipparcos observations of V505 Per only three points cover the principal eclipse. Even if model solution can fit these three points to a formal accuracy of $\sim 1 \%$ (cf. lower-right panel of Figure 9), nevertheless the massive undersampling casts many doubts on the true accuracy of derived radii. In fact, high quality and massive ground-

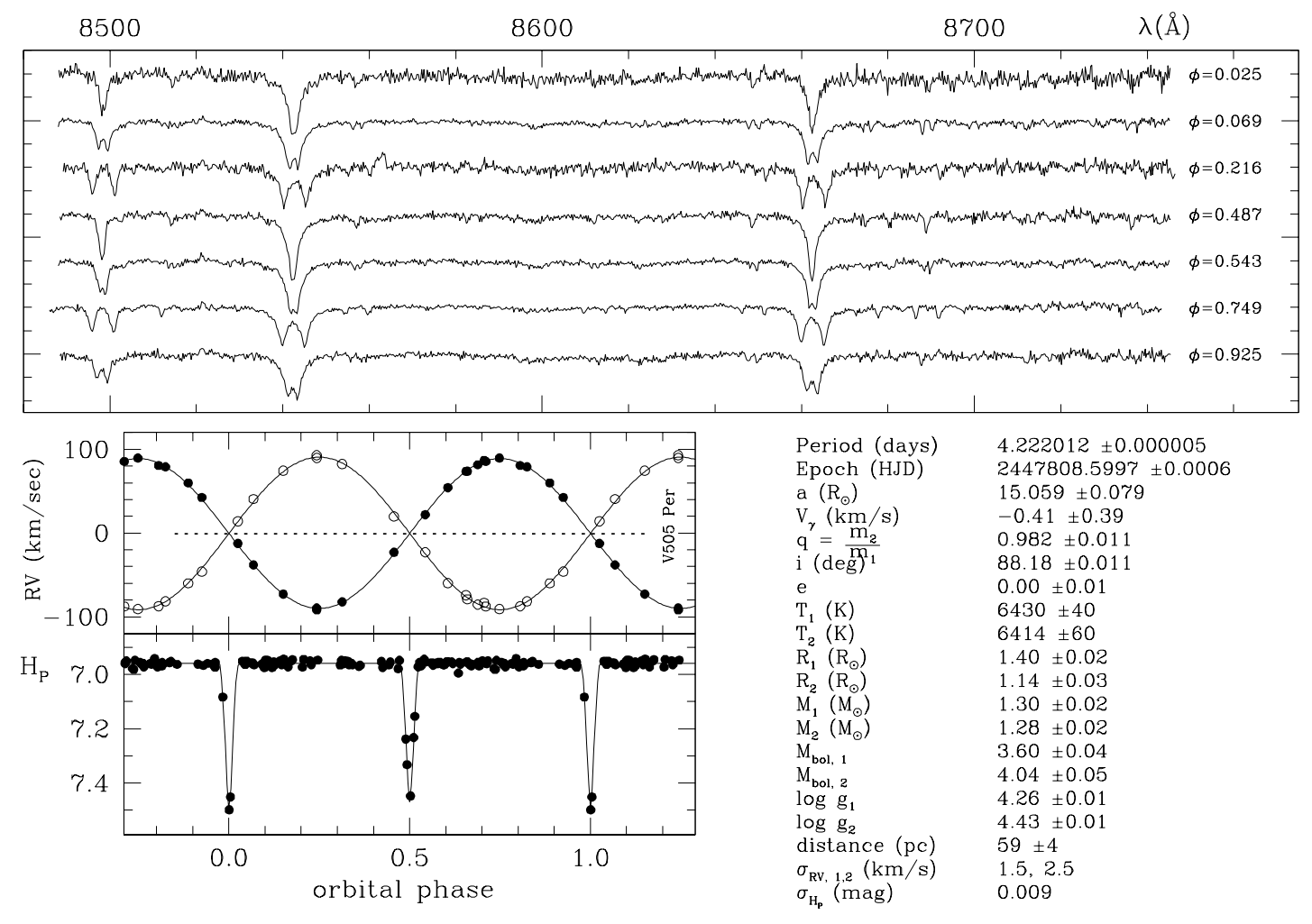

Fig. 9. V505 Per as an example of GAIA performance on eclipsing binaries (from Munari et al. 2001b). Upper panel: a sample of the recorded spectra to illustrate line splitting with orbital phase. Lower left panel: radial velocity curve and model fitting, Hipparcos $H_{P}$ photometry and model fitting. Lower right panel: orbital and physical parameters as derived from modeling of radial velocity and photometric data. 


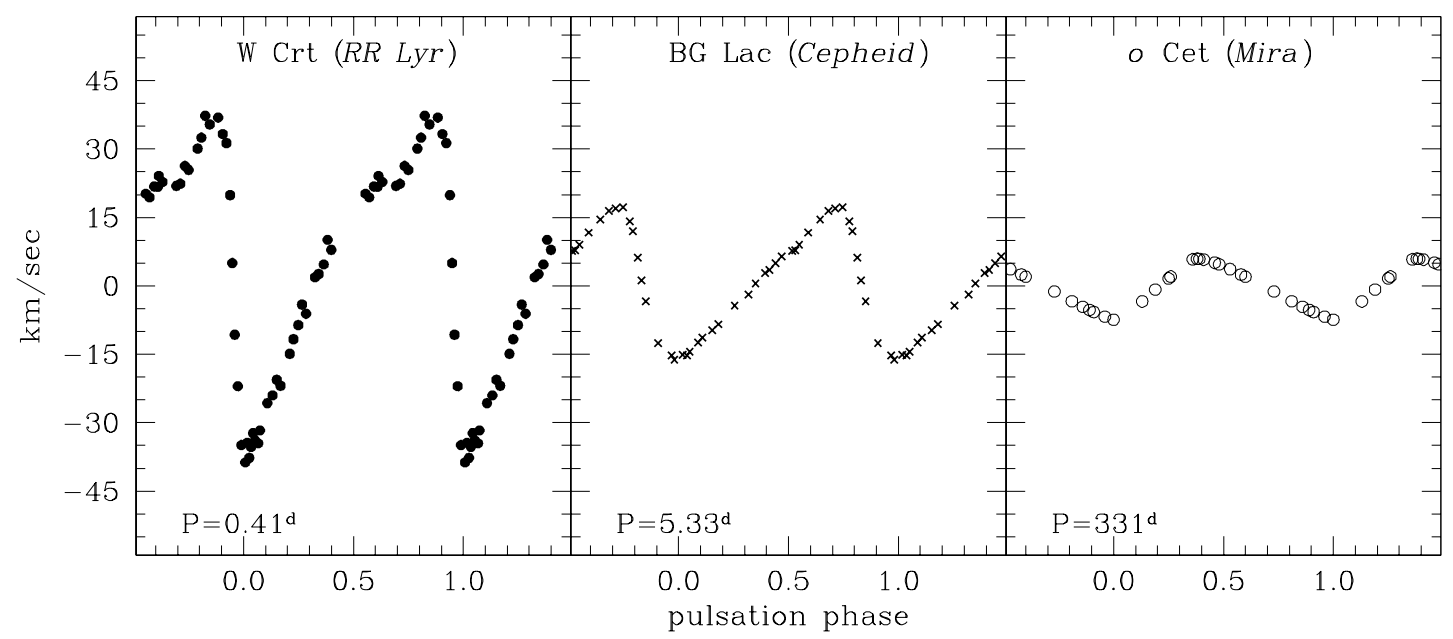

Fig. 10. Radial velocity curves of representative pulsating stars.

based observations of V505 Per by Marschall et al. (1997) fully confirm GAIA-like solution of V505 Per as reported in Figure 9 for all orbital and physical parameters but individual radii. If the sum of them is $R_{1}+R_{2}=2.54 R_{\odot}$ for the GAIA-like solution and 2.55 for Marschall et al., the individual values are 1.29 and 1.26 for the latter and 1.40 and $1.14 \mathrm{R}_{\odot}$ for M01b. Numerical experiments however show that by just doubling the number of points covering the principal eclipses in the Hipparcos lightcurve would fix the result to a much higher degree of confidence and close to the Marschall et al. findings.

It is therefore of great relevance to the broadest stellar astrophysics that a minimum number of identical photometric bands is replicated in all the three viewing fields of GAIA, so to achieve maximum density of points in lightcurve mapping of eclipsing binaries (with obvious benefit to all other types of variable objects observed by GAIA).

The high quality of the results on V505 Per shown in Figure 9 (typical of other cases investigated in $\mathrm{M} 01 \mathrm{~b}$ ) is however definitively depending upon the adopted $0.25 \AA /$ pix spectral dispersion. At such a dispersion, lines from both components are easily resolved and measured, while at the coarser $0.75 \AA /$ pix dispersion currently baselined for GAIA the lines would merge into unresolved profiles that could even hide the binary nature of the object.

\subsection{Pulsating stars}

Radial velocity mapping of pulsating stars add precious information to the effort of understanding their nature (cf. Bono et al. 1997). Non-radial pulsators manifest marked variations of line profiles that can be observed and modeled if the spectral resolution is high enough.

Spectra of template radially pulsating stars (RR Lyr, $\delta$ Cep, o Cet) are presented in M99a. The wealth of strong absorption lines, in particular Ca II, assures that accurate radial velocities can be obtained at even the lowest metallicities (RR Lyr itself has $\left[\mathrm{Z} / \mathrm{Z}_{\odot}\right]=-1.37$ ).

Pulsation curves of representative cases are given in Figure 10. Shapes and details of such curves (e.g. the glitch in W Crt at phase 0.67, or BG Lac at phase 0.52), tell a lot about interior physics of these stars. It may be easily anticipated that GAIA spectroscopic monitoring of thousands of them (compared to the very few cases investigated from the ground) will loudly impact on our understanding of stellar radial pulsations, provided the accuracy of epoch radial 
velocities will be good enough.

Semi-amplitudes for Lyrids are of the order of $\triangle \mathrm{RV} \sim 40 \mathrm{~km} \mathrm{sec}^{-1}, \triangle \mathrm{RV} \sim 15$ for Cepheids and $\triangle \mathrm{RV} \sim 7$ for Miras. So low amplitudes need high spectral resolution to be properly mapped: at $0.25 \AA /$ pix even glitches in the pulsation curves will be detected, while at $0.75 \AA /$ pix the pulsation curves of the majority of Miras will be unobservable.

\section{PECULIARITIES}

The 8480-8750 A GAIA wavelength range offers fine detection capabilities and diagnostic potential toward stellar peculiarities. Spectra of a few representative peculiar stars are presented in Figure 12 (for other types and examples see M99a) and briefly commented hereafter.

Be stars show strong and variable Paschen and He I emission lines, which profile trace conditions within the circumstellar disk. Hot mass-losing stars (P-Cyg type) present Paschen and He I lines with the characteristic emission/absorption profile that can be modeled to derive the mass-loss rate. Very wide and bright Ca II profiles (and usually weaker Paschen) trace the fast expansion of novae ejecta $\left(\mathrm{FWHM}=1280 \mathrm{~km} \mathrm{sec}^{-1}\right.$ in Nova Cyg 2001 \#1), with sub-structures correlated to and tracing the dishomogeneities and clumps in the dispersing material.

Either hot and cool pre-ZAMS objects well perform over the GAIA range. Herbig Ae/Be stars display both CaII and Paschen lines in strong emission, usually emerging from an absorption core. In cooler pre-ZAMS objects Ca II lines play the game, from the exceptional intensity in T Tau (cf. the spectrum in M99a) to the weak emission components in FU Ori.

Active atmosphere/spot stars reveal their nature by a complex cool absorption spectrum and structured emission profiles. Major bright and active areas over the stellar surface correlates with individual, radial velocity displaced (by axial rotation) components in the spectrum, as in the BY Dra spectrum in Figure 12 where emission cores in Ca II separated by $74 \mathrm{~km} \mathrm{sec}^{-1}$ are paralleled by Fe I double absorption lines separated by an identical $74 \mathrm{~km} \mathrm{sec}^{-1}$.

Spectra of interacting binary stars offer a fine and intriguing display over the GAIA spectral range. Symbiotic stars usually present strong Paschen, Ca II and weaker He I emission lines, while in some other case the naked absorption spectrum of the cool giant can be observed

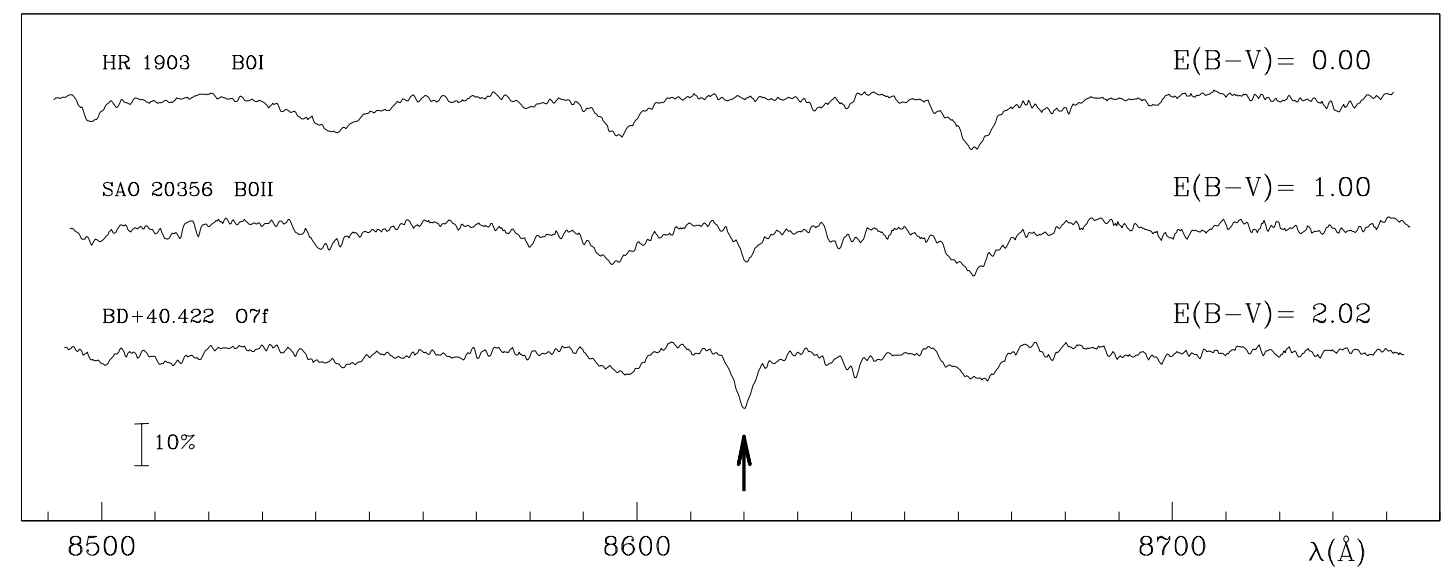

Fig. 11. The diffuse interstellar band at $8620 \AA$ (marked by the arrow) on spectra of early type stars at different reddenings. Note the increase of DIB equivalent width with reddening (from Munari 2000). 


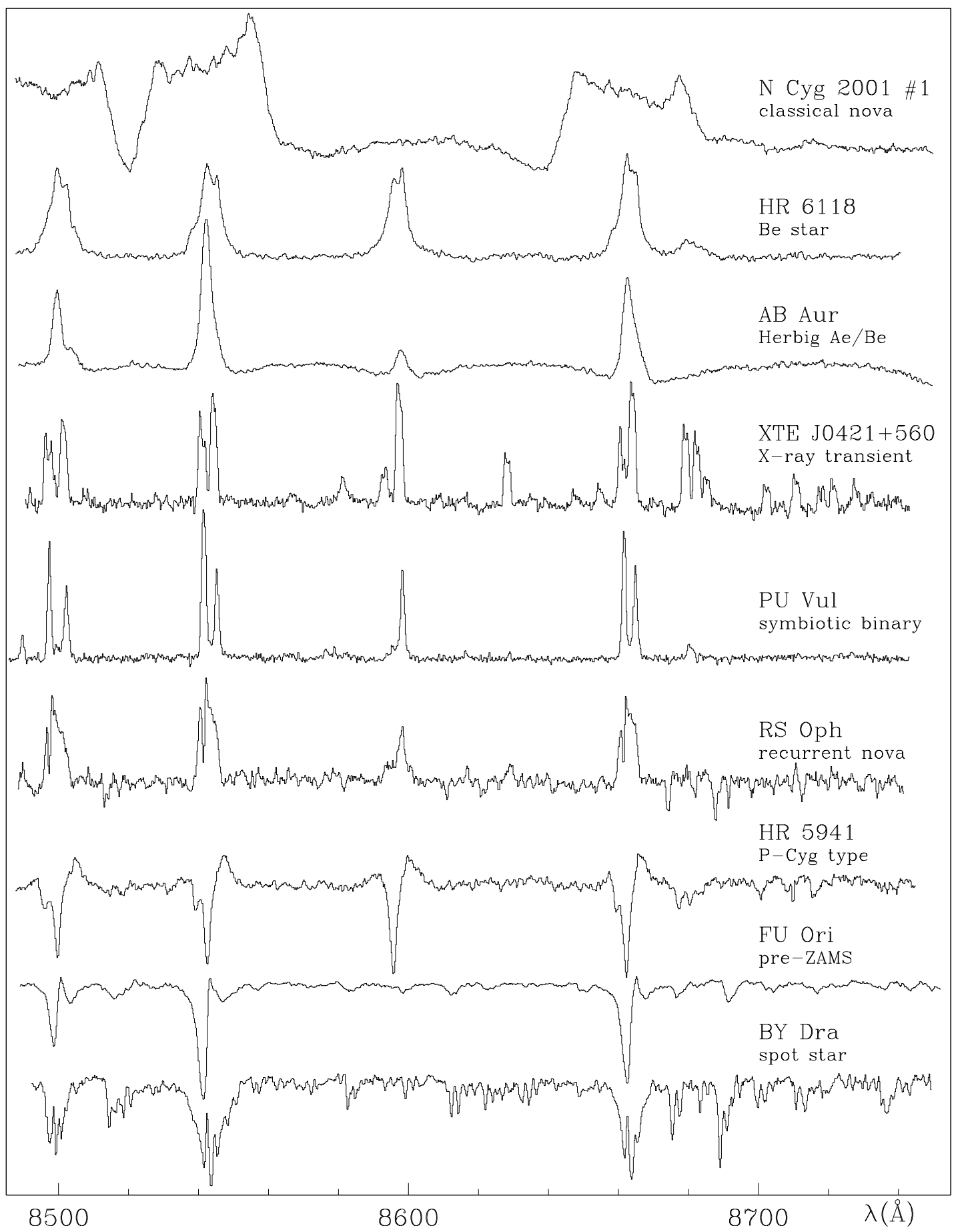

Fig. 12. Spectra of representative peculiar stars (from Munari et al. 2001c). 
without contamination from the hot companion. The same Ca II, Paschen and He I lines plus strong N I (multiplet \#1 and \#8) dominate spectra of the X-ray transient XTE J0421+560 in Figure 12, with complex line profiles indicating kinematically decoupled emitting regions. Wide, strong and multi-component, orbital-phase variable Ca II profiles stand out in the spectra of the recurrent nova RS Oph.

Finally, the interstellar medium can manifest itself in GAIA spectra. Figure 11 show the spectra of three similar hot stars affected by different amount of reddening, where absorption by the diffuse interstellar band at $8620 \AA$ clearly stands out. The equivalent width of the latter correlates surprisingly well with reddening, offering interesting opportunities for GAIA diagnostic of the interstellar medium (Munari 2000):

$$
E_{B-V}=2.69 \times E W(\AA)
$$

Efforts are currently underway (cf. Moro and Zwitter 1999) to investigate the dependence of the slope coefficient (2.69) upon the intrinsic and tri-dimensional properties of the galactic interstellar medium $\left(E_{B-V}=\alpha(l, b, \mathrm{D}) \times E W(\AA)\right)$.

\section{References}

[1] Agnolin P. 2000, degree thesis, Dep. of Astronomy, Univ. of Padova

[2] Andersen J. 1991, A\&A Review 3, 91

[3] Bono G., Caputo F., Cassisi S., Castellani, V., Marconi M. 1997, ApJ 477, 346

[4] Boschi F. 2000, degree thesis, Dep. of Physics, Univ. of Milan

[5] Boschi F., Munari U. 2001, AJ, to be submitted

[6] Carquillat J.M., Nadal R., Ginestet N., Pedoussaut A. 1982, A\&A 115, 23

[7] Castelli F., Munari U., 2001, A\&A 366, 1003

[8] Glebocki R., Gnacinski P., Stawikowski A. 2000, Acta Astron. 50, 509

[9] Jaschek C., Jaschek M. 1995, The behaviour of chemical elements in stars, Cambridge Uiv. Press

[10] Maeder A., Meynet G. 2001, A\&A 373, 555

[11] Marrese P.M. 2000, degree thesis, Dep. of Astronomy, Univ. of Padova

[12] Marschall, L.A., Stefanik, R.P., Lacy, C.H., Torres, G., Williams, D.B., and Agerer, F. 1997, AJ 114, 793

[13] Moro D., Zwitter T. 2000, in Proc. of "Molecules in Space and in the Laboratory", I.Porceddu and S.Aiello ed.s, Soc. It. Fis. 67, pag. 171

[14] Moro D., Munari U. 2000, A\&AS 147, 361

[15] Munari U. 1999a, in Proc. of "GAIA" ESA-ESTEC Workshop, V.Strayzis ed., Balt. Astron. 8, 73 (M99a)

[16] Munari U. 1999b, in Proc. of "GAIA" ESA-ESTEC Workshop, V.Strayzis ed., Balt. Astron. 8, 123 (M99b)

[17] Munari U. 2000, in Proc. of "Molecules in Space and in the Laboratory", I.Porceddu and S.Aiello ed.s, Soc. It. Fis. 67, pag. 179 astro-ph/0010271)

[18] Munari U., Tomasella L., 1999, A\&AS 137, 521

[19] Munari U., Castelli F., 2000, A\&AS 141, 141

[20] Munari U.. Agnolin P., Tomasella L., 2001a, Balt.Astron., vol. 10, November 2001 issue, in press astro$\mathrm{ph} / 0105167$, M01a)

[21] Munari U., Tomov T., Zwitter T., Milone E.F., Kallrath J., Marrese P.M., Boschi F., Prša A., Tomasella L., Moro D. 2001b, A\&A, in press (astro-ph/0105121, M01b)

[22] Munari U., Tomasella L., Marrese P.M., Boschi F., Zwitter T., Moro D., 2001c, A\&A, to be submitted

[23] Perryman, M.A.C., de Boer, K.S., Gilmore, G., Hoeg, E., Lattanzi, M.G., Lindegren, L., Luri, X., Mignard, F., Pace, O., de Zeeuw, P.T. 2001, A\&A 369, 339

[24] Straižys V. 1992, Multicolor Stellar Photometry, Pachart Pub. House, Tucson

[25] Zwitter T. 2001, A\&A, to be submitted

[26] Zwitter T, Munari U., Castelli F., 2001, A\&A, in preparation 